\title{
Implementation of the PADIS 2018 Guidelines: a National Multicenter Survey in the Adults Intensive Care Units in Mainland China
}

\section{Fangxiao Zhang}

The First Hospital of China Medical University: The First Affiliated Hospital of China Medical University

Xin Li

The First Hospital of China Medical University: The First Affiliated Hospital of China Medical University

\section{Xianyao Wan}

First Affiliated Hospital of Dalian Medical University

Jian Yu

The Second Hospital of Dalian Medical University

Hongliang Wang

Second Affiliated Hospital of Harbin Medical University

\section{Zhaoxia Yu}

Xinjiang Medical University Affiliated First Hospital

\section{Yi Yang}

Southeast University Zhongda Hospital

\section{Youzhong An}

Peking University People's Hospital

\section{Dawei Wu}

Qilu Hospital of Shandong University Qingdao

\section{Chuanyun Qian}

Kunming Medical University Second Hospital

\section{Yuan Xu}

Tsinghua University Affiliated Beijing Tsinghua Changgung Hospital: Beijing Tsinghua Changgung Hospital

\section{Qingqing Huang}

Kunming Medical University Second Hospital

\section{Mingyan Zhao}

First Affiliated Hospital of Harbin Medical University

\section{Dong Zhang}

Jilin University First Hospital

\section{Xiaochun Ma}

The First Affiliated Hospital of China Medical University

\section{Zhidan zhang ( $\square$ zhangzhidan1974@163.com)}

The First Affiliated Hospital of China Medical University https://orcid.org/0000-0003-2188-6501

\section{Research}


Keywords: guidelines, intensive care, PADIS, pain, sedation, delirium

Posted Date: May 25th, 2021

DOI: https://doi.org/10.21203/rs.3.rs-516473/v1

License: (c) (i) This work is licensed under a Creative Commons Attribution 4.0 International License. Read Full License 


\section{Abstract}

Backgroud: In the diagnosis and treatment of critical ill patients, the management of sedation, analgesia and delirium is an indispensable part. Many guidelines provide relevant suggestion, but there is still a lack of evaluation on the compliance of medical staff. This was a national multicenter survey to assess the training frequency, the familiarity of the guidelines, prevention and management of pain, agitation/sedation, delirium, immobility, and sleep disruption in the ICU.

Methods: This survey used a self-administered questionnaire online to provide data for evaluating the compliance with the PADIS guidelines. We grouped the answers that were similar in degree in order to analysis, and only complete questionnaires were included in the final analysis.

Results: The respondents from 758 hospitals across 270 cities were distributed 5763 questionnaires. The response rate was $95 \%$. Two thirds of the respondents were trained in the relevant content but only half of them had sufficient knowledge of the PADIS guidelines. The assessment frequency of pain and sedation was better than that of delirium. $83 \%$ of the respondents used physical restraint without deep sedation, $63 \%$ underwent regular treatment to improve sleep, and only $32 \%$ had opted for out-of-bed rehabilitation. Only $50 \%$ had increased family communication and early family care. The doctors provided better responses than nurses in most items, while nurses performed better in immobility, sleep disruption, and family empowerment. Also, most of the items showed better responses by University-affiliated hospitals than others, and the implementation in "large" cities with larger populations was better than that in "medium" or "small" cities.

Conclusions: Most of the Chinese medical staff complied with PADIS guidelines satisfactorily, but immobility, sleep disruption, and family empowerment could be improved further.

Trial registration: The survey was open and anonymous, and it was registered in the Chinese Clinical Trial Registry (Registration Number: NCT04217915. Registered 6 January 2020)

\section{Background}

Individualized monitoring and evaluation of sedation, analgesia and delirium, so as to protect patients' comfort and organ function, is the key to the priority in the treatment of critically ill patients. Since 2013, evidence was gathered when SCCM published the Clinical Practice Guidelines for the management of pain, agitation, and delirium in adult patients in the intensive care unit(ICU)(1), which led to the SCCM 2018 publication of the Clinical Practice Guidelines for the prevention and management of pain, agitation/sedation, delirium, immobility, and sleep disruption, known as the PADIS guidelines (2). JL Vincent proposed a strategy with early comfort using analgesia, minimal sedatives and maximal humane care(eCASH)(3). Pandharipande first proposed the evidence-based "awakening and breathing trials, choice of drugs, delirium management, and early exercise/mobility" (ABCDE) bundle in 2010(4). The Chinese Society of Critical Care Medicine also published the Chinese guidelines for sedation and analgesia in adult ICU in 2018(5). Even Several guidelines have been published and many surveys investigated the practices, the knowledge on practices in China is lacking(6). The repeated assessment of patients for pain, anticipating the sources of discomfort, and adjusting pain management strategies would allow the nurses to address the patients' needs while minimizing the risk of complications. The 2018 guidelines added recommendations addressing immobility and sleep disruption, acknowledging that these aspects of critical illness are affected by pain, sedation, and delirium(7-9). The present study aimed to provide baseline knowledge and improve the sedation practices and preferences of Chinese intensive care physicians.

Page $3 / 21$ 


\section{Methods}

This study was approved by the Committee for Ethical Affairs of the First Affiliated Hospital of China Medical University (No. 2019-331-3). The survey was open and anonymous, and it was registered in the Chinese Clinical Trial Registry (registration number: NCT04217915. Registered 6 January 2020). This survey investigated the clinicians and nurses in adult ICUs.

\section{Survey design}

We developed a set of questionnaires based on the available literature, the ICU experts, and epidemiology. The present survey was conducted using a self-administered questionnaire. A total of 6 domains were probed with a total of 34 questions. The respondents were asked to estimate the management strategy and how often these items were evaluated or intervened (never $=0-10 \%$, sometimes $=10-50 \%$, frequently $=50-90 \%$, and always $90-$ $100 \%$ ). The patients were also required to assess their knowledge of these items (none $=0-10 \%$, little $=10-50 \%$, most $=50-90 \%$, and total $90-100 \%$ ).

\section{Questionnaire}

The questionnaire was developed for the survey through "WJX" website(http://www.wjx.cn), an open platform with customers encompassing $>30000$ enterprises and $90 \%$ of domestic Universities. After the questionnaire design, the platform generated a Quick Response code(QR code) to distribute the questionnaire through WeChat. Each WeChat number was bound with a real name telephone number to ensure that the questionnaire would not be filled in repeatedly by the same person to avoid duplicate entries. The website background could view the data in real-time and control the quality of data completion. Before the questionnaire was distributed, the ICU staff from First Affiliated Hospital of China Medical University conducted a pilot test and made the necessary modifications to the content. The questionnaire was distributed through the Internet at a national conference of the Chinese Medical Association on November 18, 2020, and the filling method was explained at the conference site. We monitored the answers in real-time through the website backstage; when the number of answers was < 1 case every $6 \mathrm{~h}$, the questionnaire system was terminated .

\section{Data analysis}

In order to compare between various variables, we grouped the answers that were similar in degree, such as "never" and "sometimes" into one group and " frequently " and "always" into another group. Moreover, based on the urban population that originated from the Ministry of Housing and Urban Rural Development in 2020, we divided the cities of the respondents into three groups for subsequent analysis. Based on a survey of familiarity with the PADIS 2018 guidelines, we divided the questions of each implementation in three groups: "understanding," "assessment," and "practice" (Table 1). Only complete questionnaires were included in the final analysis. The categorical variables were expressed as frequencies and percentages. For test-retest analysis, the chi-square test for nominal data, Spearman's rho for ordinal data, and Pearson's $r$ for interval data were used. All tests of significance were two-tailed, and $P<0.05$ indicated statistical significance. The analyses were performed using Statistical Package for Social Sciences (SPSS version 26.0, IBM, NY, USA). 
Table 1

Divide each specific question into groups of " Understanding", "Assessment" and "Practice "

\begin{tabular}{|llll|}
\hline Domains & Understanding & Assessment & Practice \\
\hline Pain & Q8 & Q1, Q2, Q4, Q5 & Q3, Q7, Q9, Q10 \\
\hline Agitation/Sedation & Q4, Q6, Q7, Q8, Q9 & Q1, Q2, Q3 & Q5 \\
\hline Delirium & Q1, Q2 & Q3 & Q4, Q5 \\
\hline Immobility & None & Q2 & Q1, Q3, Q4 \\
\hline Sleep disruption & Q2 & Q1 & Q3, Q4 \\
\hline Rehabilitation & None & None & Q5 \\
\hline Family empowerment & None & None & Q6 \\
\hline
\end{tabular}

\section{Results}

The survey lasted six days from November 18-24, 2020. A total of 6041 questionnaires were distributed, and 278 invalid questionnaires were excluded(Fig. 1). The main reasons for exclusion were as follows: 1. Questionnaire forms were incomplete; 2 . Respondents were from pediatric ICU; 3 . The answers to logical questions were inconsistent. Subsequently, 5763 valid questionnaire forms were analyzed, and the response rate was $95.40 \%$. The management and practice of pain and sedation were better than those of delirium. Doctors provided better responses than nurses, but nurses performed better in immobility, sleep disruption, and family empowerment. Also, most of the items regarding University-affiliated hospitals provided better responses than non-University affiliated hospitals, and the implementation in "large" cities with larger populations was better than that in "medium" or "small" cities (Fig. 2).

\section{Respondent characteristics}

The respondents were from 758 hospitals across 270 cities encompassing the province-level administrations of mainland China (Fig. 3). Table 2 and Fig. 4 showed the number and the distribution of the respondents by province. The ratio of doctors to nurses participating in the survey was 1:1. The ratio of respondents who did or did not come from the University-affiliated Hospital was almost 1:1. Medical staff from 6 different types of ICUs participated in the survey, most were from mixed ICUs, and half had 10-20 ICU beds (Table 3 ) . 
Table 2

Number of the respondents by province

\begin{tabular}{|c|c|}
\hline Province & $N(\%)$ \\
\hline Liaoning & 734(12.74) \\
\hline Shandong & $520(9.02)$ \\
\hline Henan & $407(7.06)$ \\
\hline Sinkiang & $343(5.95)$ \\
\hline Chungking & $274(4.75)$ \\
\hline Hubei & $271(4.70)$ \\
\hline Shaanxi & $268(4.65)$ \\
\hline Tianjin & $267(4.63)$ \\
\hline Hainan & $242(4.20)$ \\
\hline Sichuan & $239(4.15)$ \\
\hline Hebei & $225(3.90)$ \\
\hline Shanghai & $210(3.64)$ \\
\hline Heilongjiang & 188(3.26) \\
\hline Guizhou & $171(2.97)$ \\
\hline Inner Mongolia & $164(2.85)$ \\
\hline Gansu & $150(2.60)$ \\
\hline Yunnan & 138(2.39) \\
\hline Ningxia & $128(2.22)$ \\
\hline Shanxi & $117(2.03)$ \\
\hline Guangxi & $97(1.68)$ \\
\hline Jiangxi & $91(1.58)$ \\
\hline Beijing & $87(1.51)$ \\
\hline Jilin & $77(1.34)$ \\
\hline Jiangsu & $75(1.30)$ \\
\hline Anhui & 72(1.25) \\
\hline Zhejiang & 68(1.18) \\
\hline Hunan & $50(0.87)$ \\
\hline Guangdong & $28(0.49)$ \\
\hline Fujian & $22(0.38)$ \\
\hline
\end{tabular}




\begin{tabular}{|ll|}
\hline Province & N(\%) \\
\hline Qinghai & $22(0.38)$ \\
\hline Tibet & $18(0.31)$ \\
\hline
\end{tabular}

Page 7/21 
Table 3

Relationship between demographic characteristics and training frequency, PADIS guidelines familiarity of 5,763 respondents

\begin{tabular}{|c|c|c|c|c|c|}
\hline \multirow[t]{2}{*}{ Characteristics } & \multicolumn{2}{|l|}{$\begin{array}{l}\text { Training Frequency } \\
\mathrm{n}=3779(66)\end{array}$} & \multicolumn{2}{|c|}{$\begin{array}{l}\text { PADIS guidelines familiarity } \\
\mathrm{n}=3350(58)\end{array}$} & \multirow[t]{2}{*}{$N(\%)$} \\
\hline & Never/sometimes & Frequently/always & None/little & Most/total & \\
\hline \multicolumn{6}{|l|}{ Gender } \\
\hline Male & 746(34.78) & $1,399(65.22)$ & 671(31.28) & $\begin{array}{l}1,474 \\
(68.72)\end{array}$ & $2,145(37.22)$ \\
\hline female & $1,238(34.22)$ & $2,380(65.78)$ & $1,742(48.15)$ & $1,876(51.85)$ & $3,618(62.78)$ \\
\hline \multicolumn{6}{|l|}{ Profession } \\
\hline Doctors & $1,123(35.98)$ & $1,998(64.02)$ & $881(28.83)$ & 2,240(71.77) & $3,121(54.16)$ \\
\hline Nurses & 861(32.89) & $1,781(67.41)$ & $1,532(57.99)$ & $1,110(42.01)$ & $2,642(45.84)$ \\
\hline \multicolumn{6}{|c|}{ Years of clinical experience } \\
\hline$\leqq 1$ year & $83(32.04)$ & $176(67.96)$ & $137(52.90)$ & $122(47.10)$ & $259(4.49)$ \\
\hline $1-3$ years & 214(31.71) & $461(68.29)$ & $343(50.81)$ & $332(49.19)$ & $675(11.71)$ \\
\hline $3-5$ years & 233(33.38) & $465(66.62)$ & $367(52.58)$ & $331(47.42)$ & $698(12.11)$ \\
\hline $5-10$ years & $590(37.02)$ & $1,004(62.98)$ & 767(48.12) & $827(51.88)$ & $1,594(27.66)$ \\
\hline$>10$ years & $864(34.06)$ & $1,673(65.94)$ & 799(31.49) & $1,738(68.51)$ & $2,537(44.02)$ \\
\hline \multicolumn{6}{|l|}{ Professional title } \\
\hline postgraduate/intern & $51(42.5)$ & $69(57.5)$ & $54(45)$ & $66(55)$ & $120(2.08)$ \\
\hline Primary & $803(32.55)$ & $1,664(67.45)$ & $1,351(54.76)$ & $1,116(45.24)$ & $2,467(42.81)$ \\
\hline Intermediate & 689(37.71) & $1,138(62.29)$ & $750(41.05)$ & $1,077(58.95)$ & $1,827(31.7)$ \\
\hline $\begin{array}{l}\text { Associate senior } \\
\text { doctor /associate } \\
\text { chief physician }\end{array}$ & $307(34.73)$ & $577(65.27)$ & 191(21.61) & 693(78.39) & $884(15.34)$ \\
\hline $\begin{array}{l}\text { Chief physician/ } \\
\text { professor of } \\
\text { medicine }\end{array}$ & 134(28.82) & $331(71.18)$ & $67(14.41)$ & 398(85.59) & $465(8.07)$ \\
\hline \multicolumn{6}{|l|}{ Education background } \\
\hline Junior college & 207(29.96) & 484(70.04) & $387(56.01)$ & $304(43.99)$ & 691(11.99) \\
\hline
\end{tabular}

$p<0.01$, Comparison between different items in "frequently/always" group; PADIS: Clinical Practice Guidelines for the Prevention and Management of Pain, Agitation/Sedation, Delirium, Immobility, and Sleep Disruption in Adult Patients in the ICU; CCU: Cardiac care unit; CSICU: Cardiosurgical intensive care unit; EICU: Emergency intensive care unit; MICU: Medical intensive care unit; NICU : Neonatal intensive care unit; NSICU: Neurosurgical intensive care unit; SICU: Surgical intensive care unit 


\begin{tabular}{|c|c|c|c|c|c|}
\hline \multirow[t]{3}{*}{ Characteristics } & \multirow{2}{*}{\multicolumn{2}{|c|}{$\begin{array}{l}\text { Training Frequency } \\
n=3779(66)\end{array}$}} & \multirow{2}{*}{\multicolumn{2}{|c|}{$\begin{array}{l}\text { PADIS guidelines familiarity } \\
n=3350(58)\end{array}$}} & \multirow[t]{3}{*}{$N(\%)$} \\
\hline & & & & & \\
\hline & Never/sometimes & Frequently/always & None/little & Most/total & \\
\hline Bachelor's degree & $1,337(35.34)$ & $2,446(64.66)$ & $1,733(48.51)$ & $2,050(54.19)$ & $3,783(65.64)$ \\
\hline Master's degree & $387(35.28)$ & $710(64.72)$ & $277(25.25)$ & $820(74.75)$ & $1,097(19.04)$ \\
\hline PHD's degree & $53(27.60)$ & $139(72.40)$ & 16(8.33) & 176(91.67) & 192(3.33) \\
\hline \multicolumn{6}{|l|}{ Type of Hospital } \\
\hline $\begin{array}{l}\text { University affiliated } \\
\text { Hospital }\end{array}$ & $1,215(37.82)$ & $1,998(62.18)$ & $1,001(39.25)$ & $1,549(60.75)$ & $2,550(44.25)$ \\
\hline $\begin{array}{l}\text { Non-University } \\
\text { affiliated Hospital }\end{array}$ & 769(30.16) & $1,781(69.84)$ & $1,412(43.95)$ & $1,801(56.05)$ & $3,213(55.75)$ \\
\hline \multicolumn{6}{|l|}{ Type of ICU } \\
\hline Mixed ICU & $1,758(33.26)$ & $3,527(66.74)$ & $2,183(41.31)$ & $3,102(58.69)$ & $5,285(91.71)$ \\
\hline CCU \& CSICU & $21(42.86)$ & $28(57.14)$ & $23(46.94)$ & $26(53.06)$ & $49(0.85)$ \\
\hline EICU & $80(47.62)$ & $88(52.38)$ & $80(47.62)$ & $88(52.38)$ & $168(2.92)$ \\
\hline MICU & $68(59.13)$ & $47(40.87)$ & $60(52.17)$ & $55(47.83)$ & $115(2)$ \\
\hline NICU \& NSICU & $27(33.33)$ & $54(66.67)$ & $38(46.91)$ & $43(53.09)$ & $81(1.41)$ \\
\hline SICU & $30(46.15)$ & $35(53.85)$ & $29(44.62)$ & $36(55.38)$ & $65(1.13)$ \\
\hline \multicolumn{6}{|c|}{ Number of beds in ICU } \\
\hline$\leqq 10$ & $512(46.29)$ & $594(53.71)$ & $559(50.54)$ & $547(49.46)$ & $1,106(19.19)$ \\
\hline $10-20$ & $1,012(34.60)$ & $1,913(65.40)$ & $1,231(42.09)$ & $1,694(57.91)$ & $2,925(50.75)$ \\
\hline $20-30$ & $320(29.36)$ & $770(70.64)$ & $418(38.35)$ & $672(61.65)$ & $1,090(18.91)$ \\
\hline$>30$ & $53(21.81)$ & 223(78.19) & 205(31.93) & $437(68.07)$ & $642(11.14)$ \\
\hline \multicolumn{6}{|l|}{ Urban Population } \\
\hline > 5 million & 459(33.24) & $922(66.76)$ & $548(39.68)$ & $833(60.32)$ & $1,381(23.96)$ \\
\hline 1-5 million & $944(33.90)$ & $1,841(66.10)$ & $1,152(41.36)$ & $1,633(58.64)$ & $2,785(48.33)$ \\
\hline$<1$ million & $581(36.40)$ & $1,016(63.66)$ & $713(44.65)$ & $884(55.35)$ & $1,597(27.71)$ \\
\hline \multicolumn{6}{|c|}{$\begin{array}{l}\text { p< } 0.01 \text {, Comparison between different items in "frequently/always" group; PADIS: Clinical Practice Guidelines } \\
\text { for the Prevention and Management of Pain, Agitation/Sedation, Delirium, Immobility, and Sleep Disruption in } \\
\text { Adult Patients in the ICU; CCU: Cardiac care unit; CSICU: Cardiosurgical intensive care unit; EICU: Emergency } \\
\text { intensive care unit; MICU: Medical intensive care unit; NICU : Neonatal intensive care unit; NSICU: Neurosurgical } \\
\text { intensive care unit; SICU: Surgical intensive care unit }\end{array}$} \\
\hline Additional file 1 . & & & & & \\
\hline
\end{tabular}

\section{Continuing education}


Nurses, University-affiliated hospital, mixed ICU, or Neonatal ICU and Neurosurgical ICU had a high percentage of training frequency which was "frequently" or "always", while MICU had the lowest percentage. Moreover, the more beds in the ICU unit or located in cities with larger populations, the higher the proportion of people "frequently" or "always" trained (Table 3). The training of sedation and analgesia management is often more than that of delirium(Fig. 5).

\section{Familiarity with different guidelines}

The gross domestic product (GDP) of each province-level administration is positively correlated with the percentage of familiarity with each guideline which was "most" or "total" (Spearman's Rho $0.48-0.6, \mathrm{P}<0.01)($ Table 4; Additional file 1). Among several different guidelines, the medical staff was most familiar with Chinese guidelines (Fig. 6). Only about 50\% was familiar with "most" or "total". A large proportion of males, doctors, and medical staff from mixed ICU, ICU with more beds, ICU of University-affiliated hospitals, or located in populated cities have a better understanding (Table 3).

Table 4

Correlation between Guidelines and GDP

\begin{tabular}{|llc|}
\hline Correlation between Guidelines and GDP & Spearman Rho & p value \\
\hline PADIS 2018 & 0.481 & 0.006 \\
\hline Chinese Guideline & 0.530 & 0.002 \\
\hline ABCDE Bundle & 0.600 & $<0.001$ \\
\hline eCASH & 0.557 & 0.001 \\
\hline $\begin{array}{l}\text { GDP: gross domestic product; PADIS: Clinical Practice Guidelines for the Prevention and Management of Pain, } \\
\text { Agitation/Sedation, Delirium, Immobility, and Sleep Disruption in Adult Patients in the ICU; }\end{array}$ \\
\hline
\end{tabular}

\section{Pain}

The critical care observation tool (CPOT) $(n=3,554 ; 62 \%)$ was most commonly used, followed by numerical rating scale (NRS) ( $n=3,417 ; 59 \%)$. Furthermore, $70 \%$ of the respondents did not use a combination of opioids and nonopioids. Only less than $50 \%$ used relaxation techniques; the widely used measures were listening to music and chatting (see Additional file 2).

\section{Agitation/sedation}

About $87 \%$ respondents evaluated the efficacy of sedation closely using Richmond agitation-sedation scale (RASS). We found that $80 \%$ of the staff rarely used bispectral index (BIS) during deep sedation. The highest familiarity of the commonly used sedative was propofol (90\%), followed by dexmedetomidine (88\%) and benzodiazepine (76\%). Only $50 \%$ of the respondents reported daily sedative interruption (DSI). About two-thirds of the respondents had sufficient knowledge of neuromuscular blockers and acquired myasthenia in the ICU. Among the tools applied, Confusion Assessment Method for the ICU (CAM-ICU) was preferred (56\%), followed by Intensive Care Delirium Screening Checklist (ICDSC) (38\%) (see Additional file 3).

\section{Delirium}


About $81 \%$ of the respondents had sufficient knowledge of the risk factors associated with delirium, while $30 \%$ knew little about the assessment progress of delirium and never used any scale or tool to assess the condition. The most popular tool was CAM-ICU (56\%). The proportion of respondents (88\%) who had used pharmacological strategy to reduce delirium is higher than those (81\%) who applied a non-pharmacological strategy. Dexmedetomidine was administered commonly (81\%), followed by centralized nursing management (77\%) and early mobilization (64\%) (see Additional file 4).

\section{Restraint}

$88 \%$ respondents stated that used physical restraint without deep sedation. They preferred restraint belts (83\%), followed by restraint gloves (48\%). About $80 \%$ respondents observed the patients' limb condition and used protective measures during restraint; also, the body position was changed intermittently. For every question in this section, nurses performed better than the doctors (see Additional file 5).

\section{Sleep disruption, rehabilitation/mobilization, and family empowerment}

$80 \%$ respondents evaluated the patients' sleep daily. About two-thirds of the respondents had a sleep-promoting protocol in their ICU; they preferred non-pharmacological intervention (92\%) than pharmacological intervention (53\%) on sleep improvement. The most popular non-pharmacological intervention was reducing sound and light stimulation at night (85\%), followed by reducing the noise (78\%) and adjusting the light in the ward periodically (66\%). Next, $73 \%$ of the respondents stated that they had conducted rehabilitation intervention for patients, $60 \%$ would adopt in-bed rehabilitation, and the remaining chose out-of-bed rehabilitation. Among the medical staff, only half would increase family communication and early family care for patients (see Additional file 6).

\section{Discussion}

This is the first nationwide study conducted to understand, assess, and practice PADIS guidelines in mainland China after it was published in 2018. The mass data had a wide coverage and reflected the true situation of medical staff's self-evaluation in adult ICU. A previous review of studies(10-12) in China presented that the medical staff had improved compliance with the guidelines, irrespective of the assessment and management of sedation, analgesia, or delirium, but there were still some shortcomings.

First, in mainland China, a satisfactory result was obtained for the education of relevant guidelines such as sedation, analgesia, and delirium. However, only about half of the people who know most of the guidelines. In a study of the barriers and enablers for implementing clinical practice guidelines in China, $27 \%$ of the Chinese medical staff reported language barriers associated with English guidelines and the impractical guidelines in the local setting (13). These observations might explain why the Chinese ICU staff was rather familiar with the Chinese guidelines. Previous studies have shown that the nurses requested additional information and training in areas such as sedation need, assessment, and management with respect to pain, delirium, and early mobilization (14, 15). The educational background and professional title of the nurses participating in the survey are lower than those of the doctors, which might be reason underlying the lack of basic knowledge. The ABCDE bundle implementation was greater in non-academic hospitals (16). However, in mainland China, the results were opposite, which might be related to the specific situation in China. According to the comprehensive ranking of hospital specialties in China released by the Hospital Management Institute of Fudan University (17), a recognized ranking board for evaluating the level of ICUs, eight out of the top ten ICUs were from University affiliated hospitals. The 
imbalance of the regional development in China affects the medical level in different regions. However, the PADIS content as the core treatment measure of ICU should not be limited by geographic or economic development. Owing to the heterogeneity of assessment among medical staff (18), uniform progress is necessary.

The respondents were more familiar with opioids than drug combination, data from many studies also suggested that the most preferred analgesic drugs were all opioids. The proption of clinicians assessing pain with tools was higher than previous, and interestingly, healthcare providers recommend CPOT and NRS as pain management tools. Some respondents reported using auricular therapy (AT) to relax the patients before an invasive procedure(19). Some prior reviews provided evidence from meta-analyses that ear acupuncture is effective for acute perioperative pain, but it had some limited evidence of effectiveness for acute pain as a stand-alone treatment(20-22).

Only 50\% respondents who implemented DSI in tubated patients. This result was lower than the data reported before $(10,16,17)$, This may due to the nouns translated into Chinese were less familiar to medical staff than spontaneous awakening trials(SATs) or spontaneous breathing trials (SBTs). Most medical staff had used tools to evaluate the efficacy of sedation, but they prefer the subjective scoring instruments to objective tools. Although it is reported that the objective sedation monitoring may reduce the total sedative use and ICU length of stay(LOS)(23), most respondents had not used BIS. Some studies suggested that the compliance were less with perceptions of high workload burden than low workload burden(24). In this survey, we did not collect data on nurse bed ratio, so whether the lower implementation rate of DSI or BIS due to fear of increasing nurse workload needs to be further explored.

Delirium is no longer a new topic in the field of critical care medicine. Numerous studies have shown that delirium is associated with the risk of mortality, ICU LOS and costs of care(25-27). The guidelines for monitoring/managing delirium have been the least utilized as previous research, but the use of assessment tools increased than before $(10,16,17,28)$. It could be concisely formulated into pamphlets or online documents so that a large number of healthcare workers could be trained with an inexpensive tool (development cost only). It is convenient to operate according to the unified process practice and conducive to ICU quality control(29). Although the guidelines did not recommend using all ICU patients and have no significant effect on key parameters $(18,30,31)$, the proportion of delirium treated with drugs, especially dexmedetomidine, remains high of its reduced incidence of delirium. This phenomenon is also relevant to the specific circumstances of the patients. In this study, we did not distinguish the specific situation of patients' self-expression. In a national multicenter cross-sectional survey currently underway, this part of the data would be collected for supplementary analysis.

The overall prevalence of physical restraint use in Chinese ICUs was higher than that reported in previous investigations $(32,33)$. Doctors had a low degree of implementation as the nurses were the actual operators of restraint and the core of evaluation. Restraint belts and restraint gloves were easier and cost less labor to physically restrain patients than restraint clothes, so the belts and gloves were prefered. Although physical restraint is necessary in some situations, but unintended consequences may occur,such as delirium, agitation, pressure ulcers, even death (34-37). So use of such devices has come under intense scrutiny and a systematic approach to reducing physical restraint use among critical illness patients is needed.

Most respondents had evaluated the patient's sleep on a daily basis but lack of a regular treatment process due to the low quality of evidence provided by the available studies(38). Despite the lack of high-quality evidence to support, the respondents still preferred non-pharmacological intervention to improve patients' sleep. Similarly, a world-wide survey stated that the patients agreed that allowing uninterrupted blocks of time to sleep was the 
intervention with the most potential to improve patient sleep in the ICU, followed by controlling environmental noise levels(39). Despite consensus that increased family visitation is necessary, but only half of the respondents chosed to do this, the reason still need further investigation. During the COVID-19 pandemic, guidelines recommended a mitigation strategy to deliver family-centered protective material (40). Presently, a new strategy of implementing regular epidemic prevention and control measures that is in agreement with the current situation should be discussed.

Nonetheless, the limitation of this study is that it is a self-assessment report. The respondents recalled the work situation and then filled in the questionaries, such that there may be a recall bias and the medical staff may overestimate their performance in the actual work or had a wrong understanding of the concepts in the questionnaires. For these aspects, a supplementary survey was conducted in the current cross-sectional study.

\section{Conclusions}

The Chinese medical staff showed good compliance with PADIS guidelines and was concerned about all aspects of the issues raised by the guidelines. In the process of implementation, some measures in line with China's national conditions were adopted. The ICUs from academic hospitals or in large cities should play a central regional role and actively organize training to improve those in peripheral hospitals.

\section{Declarations}

\section{Ethics approval and consent to participate}

This study was approved by the Committee for Ethical Affairs of the First Affiliated Hospital of China Medical University (No. 2019-331-3). The survey was open and anonymous, and it was registered in the Chinese Clinical Trial Registry (registration number: NCT04217915).

\section{Consent for publication}

All authors of the manuscript have read and agreed to its content and are accountable for all aspects of the accuracy and integrity of the manuscript in accordance with ICMJE criteria. This article is original, has not already been published in a journal, and is not currently under consideration by another journal. I agree to the terms of the BioMed Central Copyright and License Agreement.

\section{Availability of data and material}

All data generated or analysed during this study are included in this article and its supplementary information files. They are also available from the corresponding author on reasonable request.

\section{Competing interests}

The authors declare that there are no conflict of interests $₫$ we do not have any possible conflicts of interest.

\section{Funding}

Clinical research program of Chinese Medical Association(Number19030010782)

\section{Authors' contributions}


$F Z, X L, X M$ and $Z Z$ designed the research; $F Z$ and $X L$ developed the questionnaires. $X W, J Y, H W Z Y, Y Y, Y A, D W, C Q$, $Y X, Q H, M Z$ and DZ participated in the design of the study and helped distributing questionnaires and data collection from multi-centers. $X L$ contributed new reagents/analytic tools; $F Z, X L$ and $Z Z$ analyzed the data; $X M$ and ZZ participated in its design and coordination and helped to draft the manuscript; FZ and XL wrote the paper. All authors read and approved the final manuscript.

\section{Acknowledgment}

We thank the Chinese Association of Critical Care Physicians and the Chinese Society of Critical Care Medicine for their support.

\section{References}

1. Barr J, Fraser GL, Puntillo K, et al, American College of Critical Care Medicine. Clinical practice guidelines for the management of pain, agitation, and delirium in adult patients in the intensive care unit. Crit Care Med. 2013;41:263-306. DOI:10.1097/CCM.0b013e3182783b72.

2. Devlin JW, Skrobik Y, Gélinas C, et al. Clinical practice guidelines for the prevention and management of pain, agitation/sedation, delirium, immobility, and sleep disruption in adult patients in the ICU. Crit Care Med. 2018;46:e825-73. DOI:10.1097/CCM.0000000000003299.

3. Vincent JL, Shehabi Y, Walsh TS, et al. Comfort and patient-centered care without excessive sedation: the eCASH concept. Intensive Care Med. 2016;42:962-71. DOI:10.1007/s00134-016-4297-4.

4. Pandharipande $P$, Banerjee $A, M c G r a n e ~ S$, et al. Liberation and animation for ventilated ICU patients: the ABCDE bundle for the back-end of critical care. Crit Care. 2010;14:157. DOI:10.1186/cc8999.

5. Chinese Society of Critical Care Medicine. Chinese guidelines for sedation and analgesia in adult ICU in 2018. Chinese Journal of Critical Care Intensive Care Medicine (Electronic Edition). 2018;2:90-113.

6. Kai C, Yanlin Y, Hongliang L, et al. A gap existed between physicians' perceptions and performance of pain, agitation-sedation and delirium assessments in Chinese intensive care units. BMC Anesthesiol. 2021;61:1-11. DOI:10.1186/s12871-021-01286-w.

7. Tanaka LM, Azevedo LC, Park M, et al. Early sedation and clinical outcomes of mechanically ventilated patients: a prospective multicenter cohort study. Crit Care. 2014;18:R156. DOI:10.1186/cc13995.

8. Ely EW, Shintani A, Truman B, et al. Delirium as a predictor of mortality in mechanically ventilated patients in the intensive care unit. JAMA. 2004;29:1753-62. DOI:10.1001/jama.291.14.1753.

9. Timothy D, Girard JC, Jackson, Pratik P, Pandharipande, et al. Delirium as a predictor of long-term cognitive impairment in survivors of critical illness. Crit Care Med. 2010;38:1513-20.

DOI:10.1097/CCM.0b013e3181e47be1.

10. Jing W, Zhiyong P, Weihan Z, et al. A National Multicenter Survey on Management of Pain, Agitation, and Delirium in Intensive Care Units in China. Chin Med J. 2017;130:1182-8. DOI:10.4103/0366-6999.205852.

11. Jinyan X, Yunbo S, Yaqi J, et al. Perceptions, attitudes, and current practices regards delirium in China. Med (Baltim). 2017;96:e8028. DOI:10.1097/MD.0000000000008028.

12. Gong ZP, Liu XW, Zhuang YY, et al. Survey of attitudes and behaviors of healthcare professionals on delirium in ICU. Chin J Traumatol. 2009;12:328-33.

13. Yinghui J, Zimeng L, Fei $\mathrm{H}$, et al. Barriers and enablers for the implementation of clinical practice guidelines in China: a mixed-method study. BMJ Open. 2019;9:e026318. DOI:10.1136/bmjopen-2018-026328. 
14. Breanna H, Jill G, Mary FT, et al. "Sedation is tricky": A qualitative content analysis of nurses' perceptions of sedation administration in mechanically ventilated intensive care unit patients. Aust Crit Care. 2018;31:153-8. DOI:10.1016/j.aucc.2018.02.001.

15. Hilde W, Ida TB. Intensive care pain treatment and sedation: Nurses' experiences of the conflict between clinical judgement and standardized care: An explorative study. Intensive Critical Care Nursing. 2013;29:128-36. DOI:10.1016/j.iccn.2012.11.003.

16. Alessandro M, Simone P, Wesley E E, et al; Worldwide Survey of the "Assessing Pain, Both Spontaneous Awakening and Breathing Trials, Choice of Drugs, Delirium Monitoring/ Management EE/Mobility, and Family Empowerment" (ABCDEF) Bundle. Crit Care Med 2017; 45:e1111-e1122. DOI: 10.1097/CCM.0000000000002640.

17. Hospital management institute of Fudan University. the comprehensive ranking of hospital specialties in China. Available at: http://www.fudanmed.com/institute/news2019-1.aspx. Accessed April 1,2021.

18. Wang W, Li HL, Wang DX, et al. Haloperidol prophylaxis decreases delirium incidence in elderly patients after noncardiac surgery: A randomized controlled trial. Crit Care Med. 2012;40:731-9.

DOI:10.1097/CCM.0b013e3182376e4f.

19. Yinglan, Xi W, Huijuan T, et al. Auricular acupuncture with seed or pellet attachments for primary insomnia: a systematic review and meta-analysis. BMC Complement Altern Med. 2015;103:1-14. DOI:10.1186/s12906015-0606-7.

20. Yeh $\mathrm{CH}$, Chiang YC, Hoffman SL, et al. Efficacy of auricular therapy for pain management: A systematic review and meta-analysis. Evid Based Complement Alternat Med. 2014;2014:934670. DOI:10.1155/2014/934670.

21. Murakami M, Fox L, Dijkers MP. Ear acupuncture for immediate pain relief-a systematic review and metaanalysis of randomized controlled trials. Pain Med. 2017;18:551-64. DOI:10.1093/pm/pnw215.

22. Jan AL, Aldridge ES, Rogers IR, Visser EJ, Bulsara MK, Niemtzow RC. Does acupuncture have a role in providing analgesia in the emergency setting? A systematic review and meta-analysis. Emerg Med Australas. 2017;29:490-8. DOI:10.1111/1742-6723.12832.

23. Mahmood S, Parchani A, El-Menyar A, et al. Utility of bispectral index in the management of multiple trauma patients. Surg Neurol Int. 2014;5:141. DOI:10.4103/2152-7806.141890.

24. Leanne MB, ACNS-BC, Mary SD,et al. Perceptions of Workload Burden and Adherence to ABCDE Bundle Among Intensive Care Providers. Am J Crit Care. 2017;26:e38-47. DOI:10.4037/ajcc2017544.

25. Witlox J, Eurelings LS, de Jonghe JF,et al. Delirium in elderly patients and the risk of postdischarge mortality, institutionalization, and dementia: a meta-analysis. JAMA. 2010;304:443-51. DOI:10.1001/jama.2010.1013.

26. Zoë T, Terence Q, Lorn M, et al. Association between components of the delirium syndrome and outcomes in hospitalised adults: a systematic review and meta-analysis. BMC Geriatr. 2021;21:162. DOI:10.1186/s12877021-02095-z.

27. Kotfis K, Marra A, Ely EW. ICU delirium - a diagnostic and therapeutic challenge in the intensive care unit. Anaesthesiol Intensive Ther. 2018;50:160-7. DOI:10.5603/AIT.a2018.0011.

28. Salluh JI, Soares M, Teles JM, et al. Delirium epidemiology in critical care (DECCA): an international study. Crit Care. 2010;14:R210. DOI:10.1186/cc9333.

29. Elke D, Fabienne D, Deborah D, et al. The effect of an interactive delirium e-learning tool on healthcare workers' delirium recognition, knowledge and strain in caring for delirious patients: a pilot pre-test/post-test study. BMC Med Educ. 2016;17:1-10. DOI:10.1186/s12909-016-0537-0.

Page $15 / 21$ 
30. Prakanrattana U, Prapaitrakool S. Efficacy of risperidone for prevention of postoperative delirium in cardiac surgery. Anaesth Intensive Care. 2007;35:714-9. DOI:10.1177/0310057X0703500509.

31. Su X, Meng ZT, Wu XH, et al. Dexmedetomidine for prevention of delirium in elderly patients after non-cardiac surgery: A randomized, double-blind, placebo-controlled trial. Lancet. 2016;388:1893-902. DOI:10.1016/S01406736(16)30580-3.

32. Ting G, Xu W, Nan D, et al. Investigating influencing factors of physical restraint use in China intensive care units: A prospective, cross-sectional, observational study. Aust Crit Care. 2019;32:193-8.

DOI:10.1016/j.aucc.2018.05.002.

33. Cuicui Z, Di L, Qianfeng H, et al. The characteristics of ICU physical restraint use and related influencing factors in China: a multi-center study. APM. 2021;10:1198-206. DOI:10.21037/apm-20-563.

34. Burry LD, Williamson DR, Perreault MM, et al. Analgesic, sedative, antipsychotic, and neuromuscular blocker use in Canadian intensive care units: A prospective, multicentre, observational study. Can J Anaesth. 2014;61:619-30.

35. Chang LY, Wang KW, Chao YF. Influence of physical restraint on unplanned extubation of adult intensive care patients: A case-control study. Am J Crit Care. 2008;17:408-15. quiz 416.

36. Micek ST, Anand NJ, Laible BR, et al. Delirium as detected by the CAM-ICU predicts restraint use among mechanically ventilated medical patients. Crit Care Med. 2005;33:1260-5.

37. Vincent S, Danielle O, Emily C, et al. Nursing Skill Mix, Nurse Staffing Level, and Physical Restraint Use in US Hospitals: a Longitudinal Study. J Gen Intern Med. 2017;32:35-41.

38. Rongfang $\mathrm{H}$, Xiaoying J, Junmin $\mathrm{C}$, et al. Non-pharmacological interventions for sleep promotion in the intensive care unit. Cochrane Database Syst Rev. 2015;10:CD008808.

39. Biren BK, Melissa PK, Shirley FJ, et al. Perceptions and Practices Regarding Sleep in the Intensive Care Unit. A Survey of 1,223 Critical Care Providers. Ann Am Thorac Soc. 2016;13:1370-7.

40. Shadman A, Yaseen MA, Waleed A, et al. Managing ICU surge during the COVID-19 crisis: rapid guidelines. Intensive Care Med. 2020;8:1-23.

\section{Figures}




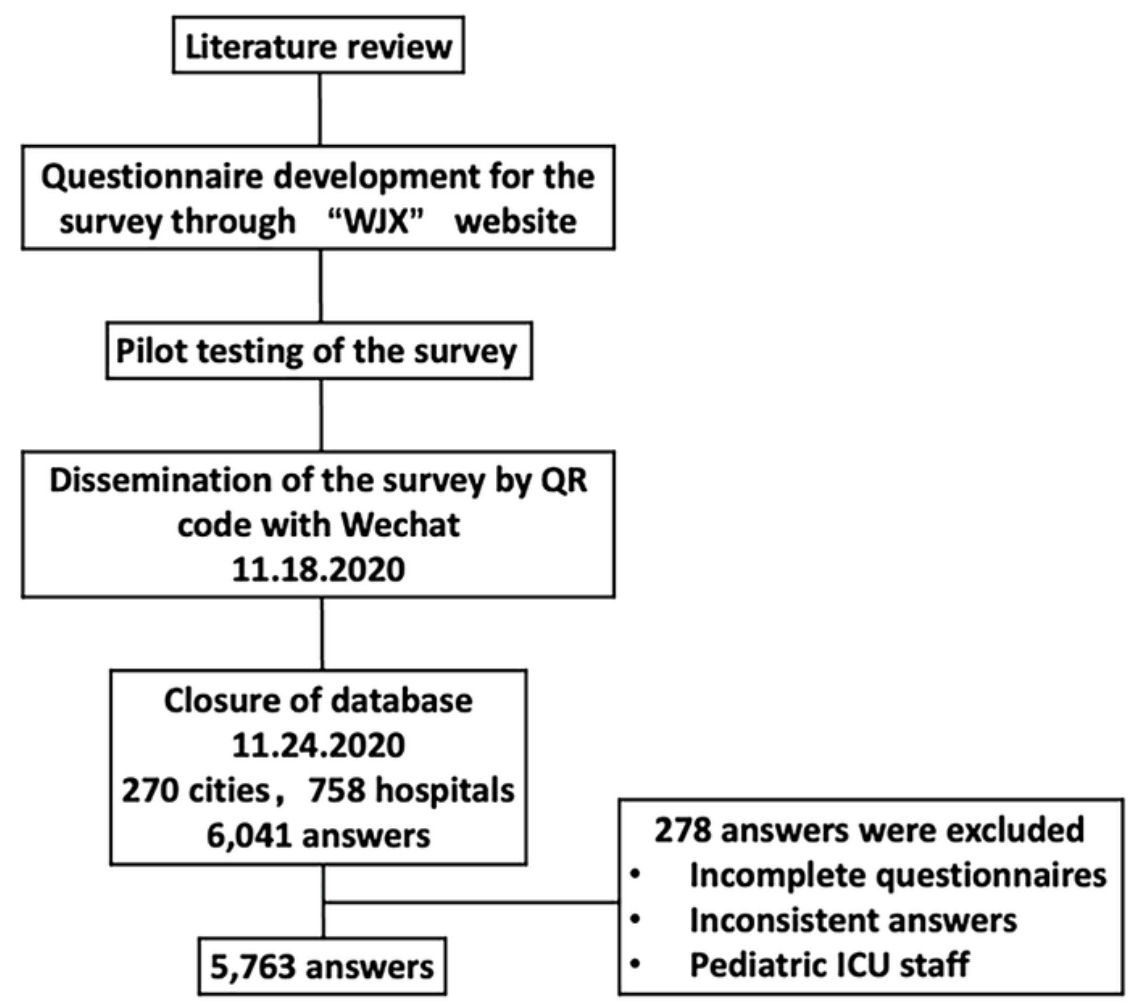

Figure 1

Flow chart of distribution and collection of questionnaires 
PAIN
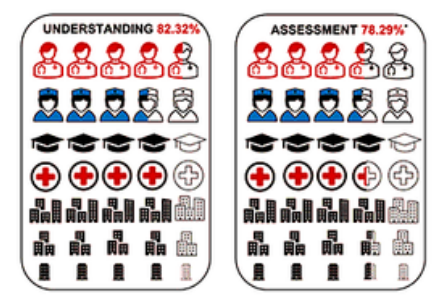

SEDATION

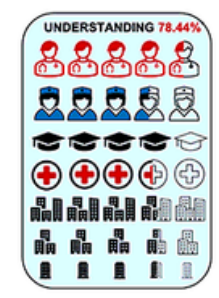

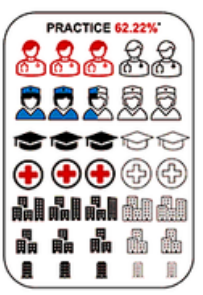

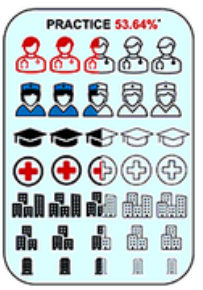

DELIRIUM

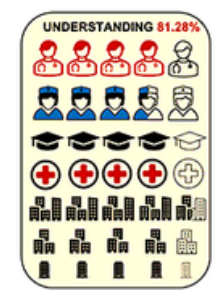

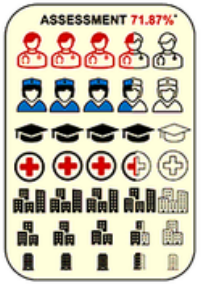

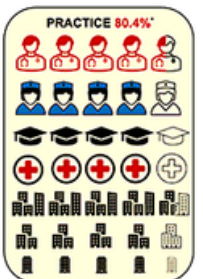

SLEEP DISRUPTION
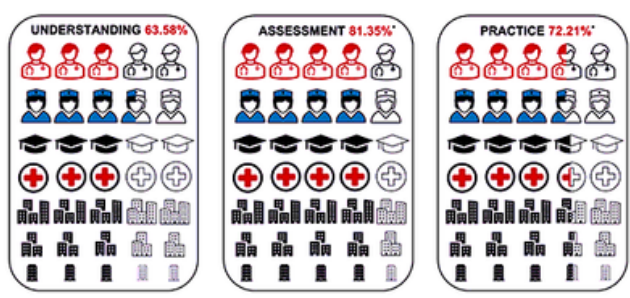

RESTRAINT
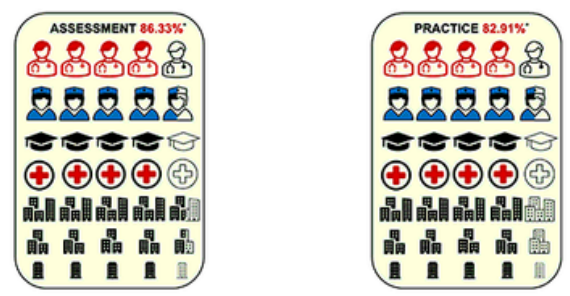

REHABILITATION

FAMILY EMPOWERMENT

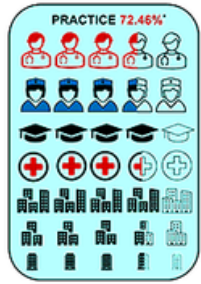

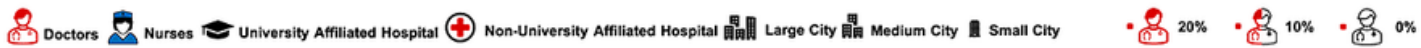

Figure 2

The understanding, assessment and practice of each items 


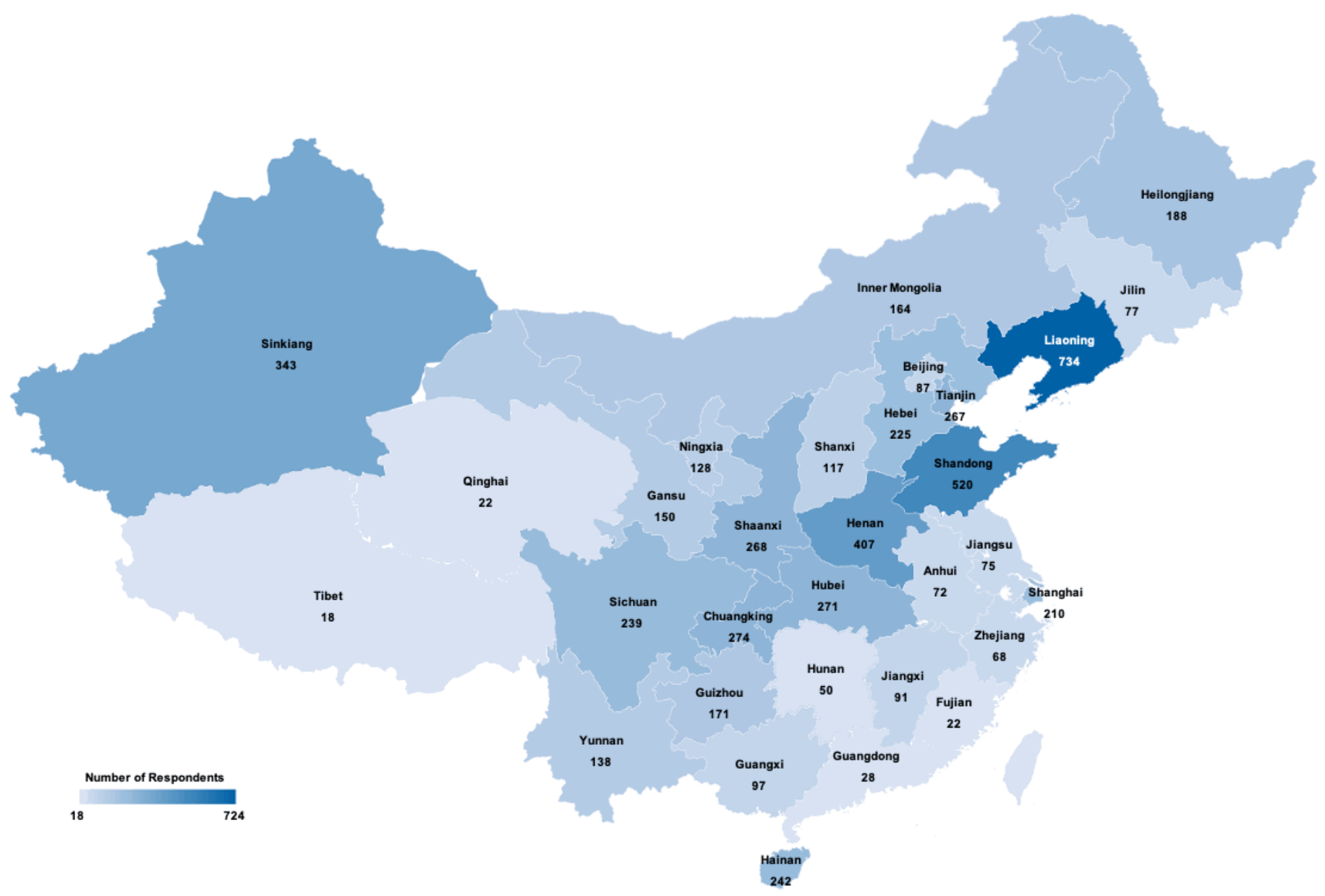

Figure 3

Province distribution of the respondents Note: The designations employed and the presentation of the material on this map do not imply the expression of any opinion whatsoever on the part of Research Square concerning the legal status of any country, territory, city or area or of its authorities, or concerning the delimitation of its frontiers or boundaries. This map has been provided by the authors. 


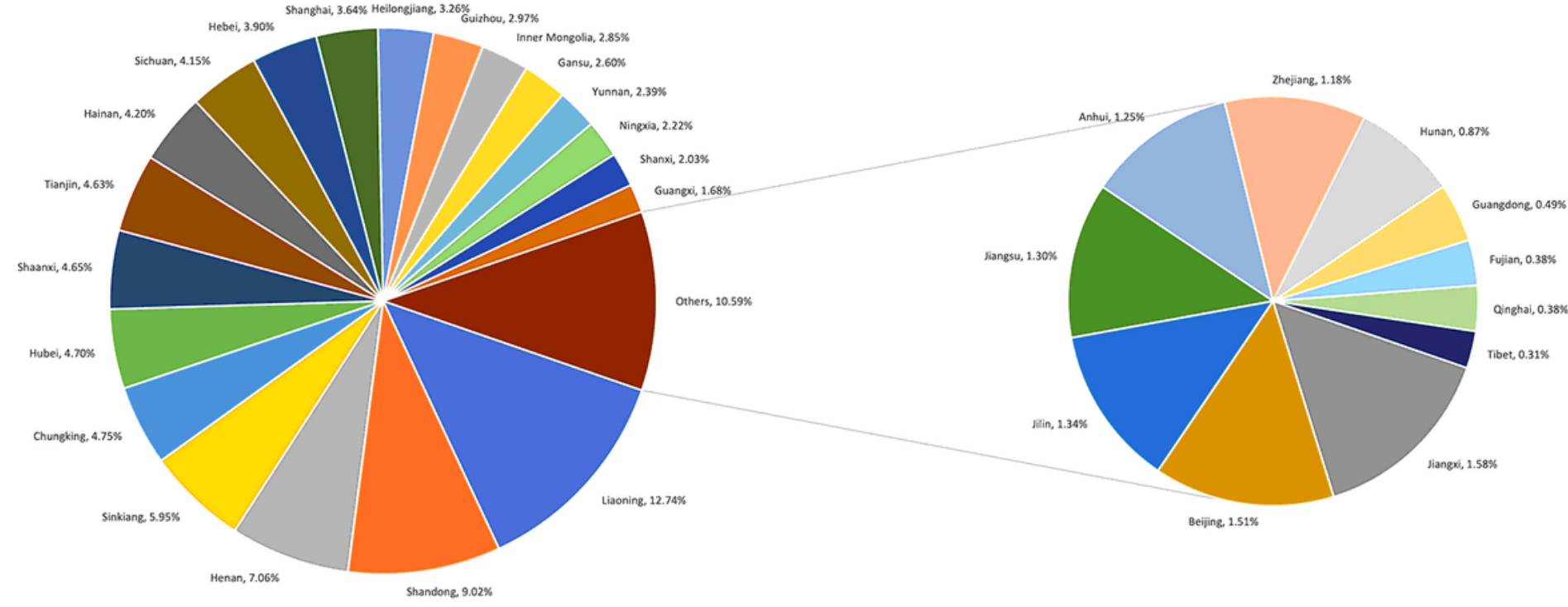

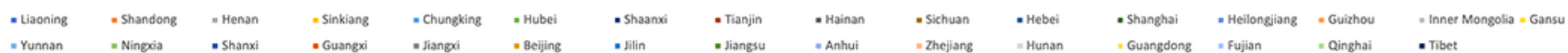

\section{Figure 4}

Numbers of the respondents by province

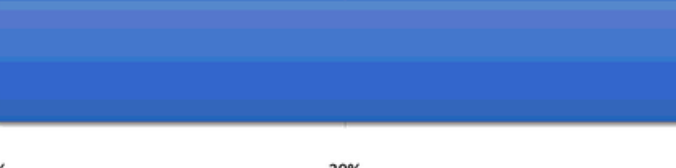

\section{Figure 5}


Ranking of the training items

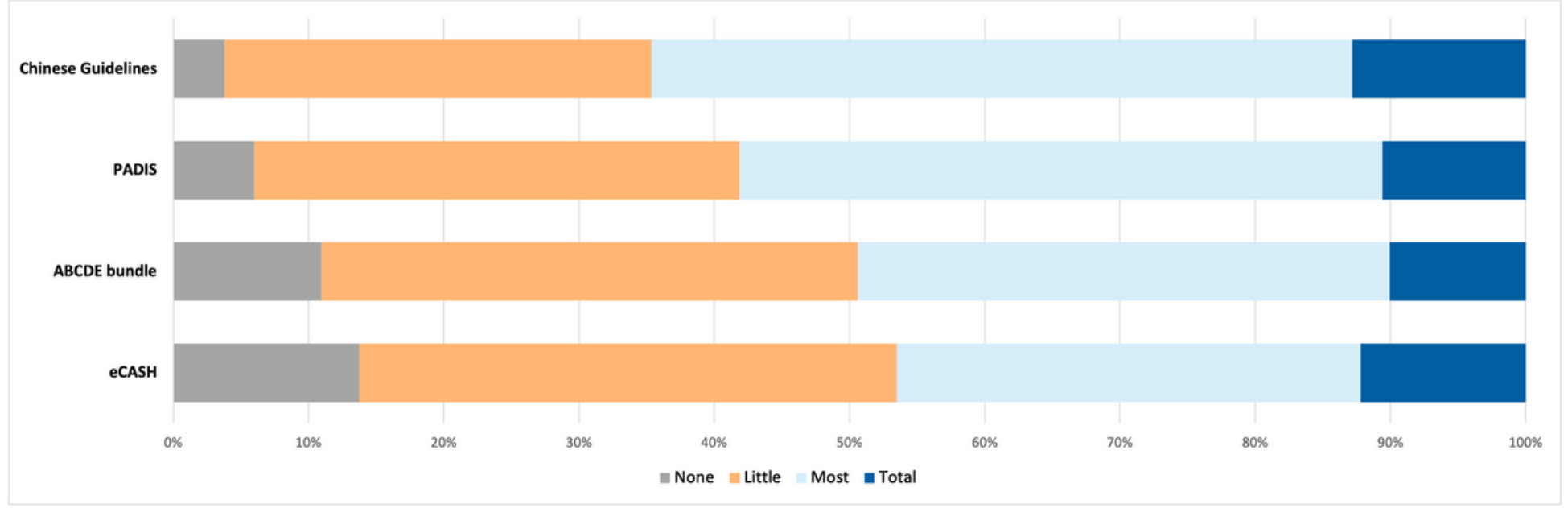

\section{Figure 6}

Ranking of the most familiar guideline

\section{Supplementary Files}

This is a list of supplementary files associated with this preprint. Click to download.

- Additionalfile1.docx

- additionalfile2.docx

- Additionalfile3.docx

- Additionalfile4.docx

- Additionalfile5.docx

- Additionalfile6.docx 